\section{WHERE TO TEACH SCIENCE}

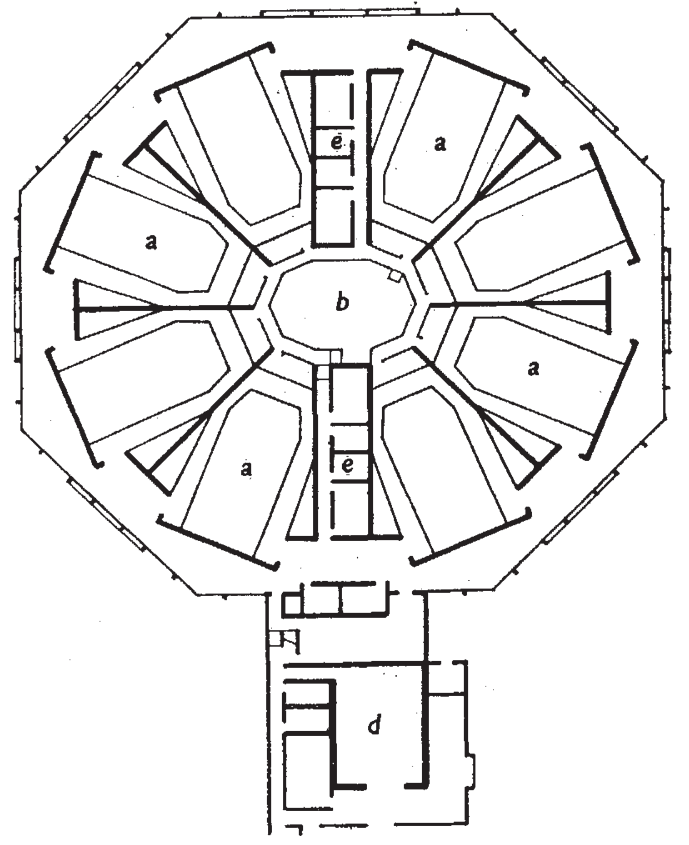

Plan for a classroom and studio building of the University of Miami, Florida. a, Auditoria, each seating three hundred people. $b$, Back projection area. From The Science Lecture Room, by Jeremy Taylor (Cambridge University Press: 60s.). The book is a planning study which examines the principles of location and design of lecture rooms in the development of university science areas.

\section{LIQUID AND SOLID HELIUM}

The Properties of Liquid and Solid Helium

By J. Wilks. (The International Series of Monographs on Physics.) Pp. xii + 703. (Oxford: Clarendon Press; London: Oxford University Press, 1967.) 150s. net.

THE torrent of scientific papers is such that physieists would be carried away beyond rescue but for the work of those few of their devoted colleagues who, by pausing from time to time to ombody their learning in monograph form, construct a series of rocks on which the rest of us may clamber from the flood and attempt to renew our perspectives for a while before diving in again. The first liquid helium rapids of the late 1930 s were stemmed in 1942 by Keesom's celebrated work, Helium, from which a whole conspectus of helium, solid, liquid, gaseous, atomic, nuclear, scientific, historic and technical, could be taken. The waters continued to rise, with the additional hazard that some navigation in Russian was called for, until Atkins in 1959 regained control of some tributaries by his book Liquid Helium. Now, twenty-five years after Keosom's book, comes Dr Wilks's The Properties of Liquid and Solid Helium.

This work will command not only respect but also gratitude from all those who have fallen under the fascination of the condensed states of helium: the respect will be shown to the author's authority, and the gratitude to the clarity with which he treats both experiment and theory. His lucid style is supported by the excellent printing and diagrams typical of the Clarendon Press. Roughly two-thirds of the book is devoted to liquid helium four, one sixth to liquid helium three, and the remaining sixth to the solid phases. As is proper, tho author's particular interest in zero sound is allowed to detain us a little when we read about helium three.

One of the conspicuous features of the book is the gathering together and presentation of introductory material necessary for an understanding of the theory. Thus the author takes care that the requisite ideas of fluid mechanics, statistical mechanics and quantum mechanics are explicitly laid out ready for use. He also offers a quite invaluable chapter on phonons in crystalline solids, by way of ixitroduction to the excitation theory of liquid helium II.

It is only to be hoped that the rather high price of this book will not prevent it from being available to the many scientists to whom it has so much to offer.

\section{V. OSBORNA}

\section{RELATIVITY AND SOLUTIONS}

Relativistic Hydrodynamics and Magnetohydrodynamics Lectures on the Existence of Solutions. By Andre Lichnerowicz. (The Mathematical Physics Monograph Series.) Pp. ix + 196. (New York: W. A. Benjamin, Inc., 1967.) $\$ 9.50$.

THIs book is addressed to a restricted audience; it is concerned with the mathematical study of the equations of relativistic hydrodynamics. Its origin lies in a course of lectures given to graduate students at the Centre of Advanced Study in Dallas at the end of 1965. The book is, in essence, the reproduction of a set of mathematical lecture notes with an almost complete absence of physical interpretation.

Familiarity with relativistic hydrodynamics is assumed and this involves a working knowledge of relativity. hydrodynamics and the theory of partial differential oquations.

In a fluid which is hydro- and thermo-dynamically perfect the treatment yields three systems, gravitational waves, hydrodynamic waves and stream lines, and the problem is extended by introducing an electromagnetic field and a fluid which is charged but has a vanishing electrical conductivity. The more difficult case of relativistic magnetohydrodynamics assumes inductive effects on a perfect and infinitely conducting fluid. This leads to Alfven, hydrodynamic and relativistic shock waves.

JohN PAIN

\section{MASS SPECTROMETRY}

\section{Mass Spectrometry of Organic Compounds}

By Herbert Budzikiewicz, Carl Djerassi and Dudley $H$. Williams. (Holden-Day Series in Physical Techniques in Chemistry.) Pp. xv +690 . (San Francisco and London: Holden-Day, Inc., 1967.) \$19.75.

This is both a very valuable and an interesting book, for several reasons. The coverage of the organic compounds discussed is very wide, comprising most simple classes of materials possessing functional groups, including the alcohols, aldehydes and ketones, ethers and other oxygen-containing molecules; their sulphur analogues: amines, amides, cyanides and other nitrogenous systems; halides, as well as the parent hydrocarbons; organic phosphorus compounds and even a comprehensive, if brief, account of organo-metallic systems.

Moreover, this book is written from a distinet point of view which is clearly stated at the outset (page 9, first paragraph et seq.); namely, that of charge locadization. This is an important unifying concept which is currently of considerable interest. The views of these authors with whom this theory is already associated as a consequence of an earlior work (Interpretation of Mass Spectra of Organic Compounds) are most timely, although the simplicity of the idea is somewhat eroded by specific com. pounds, notably among oxygen-containing molecules (for example, page 100, line 6; page 133, line 9; page 180 , fourth paragraph). 